\title{
Update on Veterinary Viral Vaccines: A Review
}

\section{Gadissa L and Yune N*}

School of veterinary medicine, College of agriculture and veterinary medicine, Jimma University, Ethiopia

*Corresponding author: Nesradin Y, 2School of veterinary medicine, College of agriculture and veterinary medicine, Jimma University P.O. Box 307, Jimma, Ethiopia, Email: Nesradin.90@gmail.com

\section{Review Article}

Volume 3 Issue 3

Received Date: August 07, 2018

Published Date: September 07, 2018

\section{Abstract}

Vaccine has made a very significant impact on the control of viral diseases in both humans and animal species. Worldwide eradication of small pox and rinderpest and drastic reduction in other infection disease are confirming to the fact that vaccination is the most feasible and cost effective strategy for prevention, control and eradication of infectious disease. Veterinary science has made a significant contribution to the field of vaccine research and development. Among the numerous of infectious diseases in animals, those of viral etiology account for a high burden of cases and they are the most relevant from a veterinary perspective. So, vaccination is the most feasible means that has to be implemented for controlling and eradicating these diseases. The viral vaccines used in veterinary medicine generally categorized into 1 of 3 categories: inactivated vaccines (in which antigens are typically combined with adjuvants); live attenuated vaccines; and recombinant technology vaccines, which may include subunit antigens or genetically engineered organisms. The majority of vaccines available today rely either on attenuation (weakening) techniques or inactivated (killed) forms of the infectious agent. Even though many vaccines are available and vaccine producing technologies are existed, several viral diseases have no vaccines yet and there are also limitations even on existing vaccines. Therefore, the objective of this seminar paper is to overview the development of veterinary viral vaccines and challenges and opportunities existing in the process of its development. To be profitable from the veterinary viral vaccines the challenging factors for the development of the vaccines should be managed. In addition, the novel vaccine technologies should be encouraged because they can fill the limitations of conventional live and killed vaccines.

Keywords: DNA vaccine; Killed vaccines; Live vaccines; Recombinant viral vaccines; Viral disease

\section{Introduction}

The term "vaccine" comes from the Latin term "vacca," meaning cow, was first coined by Edward Jenner to describe the inoculation of humans with cowpox virus to confer protection against the related human smallpox virus. Jenner used the cowpox virus against the smallpox virus in one of his patients. He further conducted many experiments and confirmed that exposure to cowpox could indeed safely protect human against smallpox. This illustrated the close relationship between human and animal infectious disease sciences [1]. In modern science 
vaccine can be defined as all products designed to stimulate active immunization of animal against disease [2].

Vaccination has made an enormous impact on the control of viral and bacterial diseases in both humans and animal species. The underlying mechanism of protection induced by vaccines is generally considered to be based on the imprinting of the immune system by pathogenspecific molecules presented in a none poorly infectious form present in the vaccine preparation, followed by the activation of an immune memory response against these molecules upon natural exposure to the live and virulent pathogen. The nature of immunological memory determines the duration of this vaccine-induced immunity and can last for the lifetime of the host [3].

Worldwide eradication of small pox and rinderpest and drastic reduction in other infection disease are attesting to the fact that vaccination is the most feasible and cost effective strategy to prevent, control and eradication of infectious disease. In food animals, the main goal of vaccination is to increase the immunity in the population rather than in the individual, and herd immunity must be considered as an indicator of the efficacy of vaccination [4]. The presence of immune animals with in the herd will reduce the probability of encountering the infection for susceptible animal and will increase the resistance of the immunized herd as a group [5].

Numerous conventional live and inactivated viral vaccines have been produced by animal health companies and have been used for many decades in routine vaccination protocols for both companion and production animals. Although wide spread use of this vaccine has contributed considerably to the improvement of public health and animal throughout the world, they have serious short comings and are far from perfect. Conventional vaccines are expensive to produce, require adjuvant and multiple inoculations to induce optimal immunity, may interfere with maternal antibodies, and consequently confer little or no protection in neonates [6]. The evolution of new technology in the field of molecular biology and immunology has further more had a large impact on the development of new vaccine strategies that fill the limitation of conventional vaccines [7].

Among the plethora of infectious diseases in animals, those of viral etiology account for a high burden of cases and are among the most relevant from a veterinary perspective. In fact, approximately half of the most important animal diseases are caused by viruses, according to the OIE's classification for terrestrial and aquatic notifiable animal diseases [8]. These diseases have several impacts by affecting animal welfare, reducing productivity, and in the worst cases, seriously undermining the economy of nations. In some cases, livestock or animal pathogens can also cause disease in humans. So, vaccination which is the best means to control and eradicate them have to be into attention [8].

Preventing transmission of infectious diseases at the animal-human interface is important for protecting the world population from both epizootics and pandemics, constituting the basis for the "One Health" concept $[9,10]$. Because there are no broad-spectrum antiviral pharmaceuticals available, hygienic measures to limit exposure and vaccination are the only means to prevent or control viral infections [11]. Even though vaccination is the major promising measure to control and prevent viral diseases, there is no satisfactory awareness regarding the veterinary viral vaccines and its development at the required level. Therefore, the objective of this seminar paper is to review the development of veterinary viral vaccines, and challenges and opportunities existing in the process of its development.

\section{Literature Review}

\section{Historical Development of Vaccines}

Vaccine was born with Edward Jenner's discovery of small pox vaccine that changed the world of medicine forever. He discovered that immunization with antigenetically related but less virulent virus protects against a more virulent virus. Vaccines are an active immunization technique where an attenuated or harmless form of a pathogen is administrated to an individual, protecting that individual against later exposure to the same pathogen [12].

Veterinary science has made a significant contribution to the field of vaccine research and development. Indeed, many of the new vaccine technologies available today found their first commercial application within veterinary medicine. The majority of vaccines available today rely either on attenuation (weakening) techniques or are inactivated (killed) forms of the infectious agent. However, both approaches have their limitations and potential associated problems such as safety, stability, efficacy and economy of manufacture. Due to this and the need to tackle emerging diseases, scientists have increasingly turned their attention towards the 
development of new technology vaccines. These novel vaccines can also be broadly separated into live and nonlive approaches [11].

Due to problems in obtaining sufficient quantities of natural subunit proteins, it became the goal of many researchers to produce large quantities of those proteins in a sufficiently pure form to generate safe and effective vaccines using recombinant DNA technology. This meant that foreign genes could be inserted into expression vectors and then introduced into cells which acted as "production factories" for the foreign proteins encoded for by those genes. In many cases the technology has provided a relatively inexhaustible and cheap source of protein for vaccination studies. Recombinant subunit vaccines have now been produced in bacterial cells, yeast, insect cells, mammalian cells, plant cells and microalgae. These proteins are presented to the host in the form of micelles, virosomes, liposomes, nanoparticles or virus like particles [13].

\section{Importance of Veterinary Vaccines}

Safe and efficient food production: Veterinary vaccines are used in livestock production and poultry rearing to maintain animal health and to improve overall production. This would help to feed the growing population with high quality proteins and other essential nutrients. FAO High-Level Expert Forum reported in September 2009 that the world population will have reached about 9.1 billion in the next 2050. In order to feed a projected world population in the next 2050 , the overall food production will need to increase by $70 \%$ between 2005/07 to 2050 [14]. Therefore, vaccines that preserve animal health and improve production are important components in meeting this need [15].

Control of zoonotic diseases: Vaccines have a big role in controlling and reducing the incidence of zoonotic diseases such as anthrax, brucellosis, avian influenza, Rift Valley fever, rabies etc. Without rabies vaccines, it is unlikely that families would be willing to keep cats and dogs as pets. Recombinant vaccinia-vectored rabies vaccines have also been used successfully in baits for oral vaccination campaigns to reduce the incidence of rabies in wild animals [16]. Veterinary vaccines for the following zoonotic diseases have been, or could be, used to control infections in animals, thereby it reduces transmission of the infectious diseases like Rabies, Brucellosis, Leptospirosis, Influenza, Rift Valley fever, Nipah and Hendra, Japanese encephalitis, and Q fever to people [17].

Control of emerging and exotic diseases of animals and people: Emerging and exotic animal diseases are a growing threat to human and animal health and jeopardize food security. Increases in human and animal populations, with accompanying environmental degradation and globalized trade and travel, enhance opportunities for transfer of pathogens within and between species. The resulting diseases pose enormous challenges now and for the future. In most of the world, increased demand for animal protein has resulted in intensified commercial food animal production and/or expanded "backyard" production. Both types of production present unique challenges for disease emergence and control. Emerging zoonotic diseases of both food and companion animals are a major threat to public health. It is inevitable that the world will continue to experience emerging disease outbreaks in the coming decades. Rapid development of animal vaccines can play a key role in controlling and helping to solve public health crisis of emerging or re-emerging pathogens of zoonotic significance [18].

Reduction of the need for antibiotics: Veterinary vaccines reduce the need for antibiotics to treat infections in food producing and companion animals. Producers may choose either vaccines or antibiotics to control some diseases based on cost, and they will use the approved control method that is most cost effective, if both options are available. If regulatory requirements for a biologics company to obtain and maintain a license to produce the vaccine were to increase, then the cost of the vaccine would increase and producers would opt to use less vaccine and more antibiotics. Affordable and available vaccines reduce reliance on antibiotics for animal health [15].

Reduce antibiotic resistance: There are increasing concerns related to antibiotic resistance associated with the extensive use of antibiotics in veterinary and human medicine [19]. The global increase in disease caused by drug-resistant bacteria, due to overuse and misuse of antibiotics, is a major public health concern. It is more difficult and costly to treat antibiotic-resistant infections and animals do not always recover. So, making better use of existing vaccines and developing new vaccines are important ways to tackle antibiotic resistance [20].

For food safety: Recently, vaccines have been developed to reduce the shedding of organisms that cause food borne diseases in people. These vaccines typically do not improve the health of the vaccinated animal, but they reduce the shedding of pathogens that may contaminate animal products for human consumption. Vaccines present a clear advantage over therapeutic molecules in that they do not leave residues. So, animal production will be free from antibiotic residue if vaccine is used as a disease prevention method instead of antibiotics for animal treatment [15]. 


\section{Types of Viral Vaccines}

Viruses (especially RNA viruses) are highly variable. Consequently, many of the existing viral vaccines are often unable to cope with the prevailing strains in the field, and new ones have to be generated from field strains with new outbreaks [21]. Numerous conventional live and inactivated viral vaccines have been produced by animal health companies and have been used for many decades in routine vaccination protocols for both companion and production animals [11]. The viral vaccines used in veterinary medicine generally fall into 1 of 3 categories: inactivated vaccines (in which antigens are typically combined with adjuvants); live attenuated vaccines; and recombinant technology vaccines, which may include subunit antigens or genetically engineered organisms [22].

Conventional attenuated viral vaccines: Live attenuated vaccine is a vaccine prepared from microorganism whose disease-producing ability has been weakened but whose immunogenic property have not or it can be defined a vaccine that induces an immune response, which more closely resembles that of a natural infection, than that elicited by killed vaccines, as the organisms contained therein actively reproduce until held in check by the recipient's own antibodies thus often conferring lifelong immunity [23]. As with the first vaccine for human smallpox, most live veterinary viral vaccines induce mild infections with live organisms derived from non-target hosts or attenuated through passage in different cell line cultures or chicken embryos [24]. As the live organism can still infect target cells, these vaccines can replicate and induce both cellular and humeral immunity and generally do not require an adjuvant to be effective. Live products also offer the advantage of ease of administration, potentially in drinking water, intranasally, intraocularly, etc. However, they can pose a risk of residual virulence and reversion to pathogenic wild types as well as provide a potential source of environmental contamination. The high rate of spontaneous mutations of RNA viruses increases the risk for reversion to virulence. Safe live viral vaccines are therefore likely to require a number of attenuating mutations distributed throughout the genome [25].

Conventional inactivated or killed vaccine: Inactivated or killed viral vaccines are generally more stable and do not pose the risk of reversion to virulence as compared to live vaccines (Table 1), but their inability to infect cells and activate cytotoxic $\mathrm{T}$ cells makes them much less protective [26]. Consequently, they generally require strong adjutants and several injections to induce the required level of immunity and are usually effective in controlling only clinical signs rather than infection. Inactivated adjuvanted vaccines also pose a greater risk of causing autoimmune diseases, allergic disorders, and vaccine injection site sarcomas [26]. Viral inactivation is commonly achieved through heat or chemicals (e.g., formaldehyde, thiomersal, ethylene oxide, and Bpropriolactone). The higher production cost and need for adjutants make these vaccines more expensive to manufacture. Inactivated viral vaccines for a wide range of viral diseases have been available for several decades [27] and are still being developed for some recently emergent diseases. For example, a one-dose inactivated porcine circovirus type 2 (PCV2) vaccine has recently been licensed in the United States for the prevention of post weaning multi-systemic wasting syndrome in pigs. Much of the recent research in this area has concentrated on the development of improved adjuvanted formulations to overcome the effects of maternal antibodies on young animals [23].

\begin{tabular}{|c|c|c|}
\hline Types of Vaccines & Advantages & Disadvantage \\
\hline \multirow{6}{*}{ Live vaccines } & $\rightarrow$ Mode of action similar to natural infection & $\rightarrow$ Reversion to virulence \\
\hline & $\begin{array}{l}\rightarrow \text { Multiply in host-induce range of } \\
\text { immune responses }\end{array}$ & $\rightarrow$ Contaminating viruses \\
\hline & $\rightarrow$ Duration of immunity usually long- lasting & $\rightarrow$ Interference by other agents and passive antibody \\
\hline & \multirow{3}{*}{$\rightarrow$ No adverse side effects to foreign protein } & $\rightarrow$ Storage problems \\
\hline & & $\rightarrow$ Possible production of latency \\
\hline & & $\rightarrow$ Possible induction of abortion \\
\hline Killed vaccines & $\rightarrow$ Quite stable & $\begin{array}{l}\rightarrow \text { Require large amounts of antigen/may not contain } \\
\text { protective antigens if these are secreted proteins }\end{array}$ \\
\hline
\end{tabular}




\section{Open Access Journal of Veterinary Science \& Research}

\begin{tabular}{|c|c|c|}
\hline & \multirow{6}{*}{$\rightarrow$ Easy to produce } & $\rightarrow$ Reactions develop to foreign protein or adjuvants \\
\hline & & $\begin{array}{c}\rightarrow \text { Immunity is usually short-lived multiple } \\
\text { doses required }\end{array}$ \\
\hline & & $\rightarrow$ Don't produce local immunity \\
\hline & & $\rightarrow$ May not inactivate all of the agent \\
\hline & & $\begin{array}{l}\rightarrow \text { Other agents may be present which } \\
\text { are resistant to inactivating agent (prions) }\end{array}$ \\
\hline & & $\rightarrow$ Induction of aberrant disease \\
\hline
\end{tabular}

Table 1: Advantages and disadvantages of live versus killed conventional vaccines.

Source: [28].

Recombinant viral vaccines: Traditional approach of inactivated or live-attenuated vaccine immunization has resulted in impressive success in the reduction and control of infectious disease outbreaks. However, many pathogens remain less amenable to deal with the traditional vaccine strategies, and more appropriate vaccine strategy is in need. Recent discoveries that led to increased understanding of viral molecular biology and genetics has rendered the use of viruses as vaccine platforms and as potential anti-cancer agents [29]. Recombinant viral vaccines fall into three basic categories: recombinant inactivated vaccines (subunit vaccines), live genetically modified organisms or live viral vector vaccines and genetic (DNA) vaccines [30].

\section{$>\quad$ Subunit Vaccines}

This technology requires the use of a segment or parts of the viral protein to induce specific immune response. Instead of the entire microbe, subunit vaccines include only the antigens that best stimulate the immune system. These vaccines are derived from pathogen protein or polysaccharide, or in some cases, these vaccines use epitopes-the very specific parts of the antigen that antibodies or $\mathrm{T}$ cells recognize and bind to. Because subunit vaccines contain only the essential antigens and not all the other molecules that make up the microbe, the chances of adverse reactions to the vaccine are lower [31].

Identification of the protective viral antigens potentially allows their isolation and/or recombinant production so that they can be administered as safe, nonreplicating vaccines. However, as isolated antigens generally induce poor protective immunity, especially Tlymphocyte activation are too weak, subunit vaccines usually require repeated administration with strong adjuvants, making them less competitive.
Notwithstanding these limitations, there are some examples of effective subunit vaccines [32].

A recombinant subunit vaccine has been made for the hepatitis B virus. The gene of hepatitis B virus that code for the required antigens should be inserted into the baker's yeast. The yeast then produced the antigens, which are collected and purified for use in the vaccine. Research is continuing on a recombinant subunit vaccine against hepatitis $C$ virus [31]. PCV2 is considered to be the major pathogen in the etiology of post weaning multisystemic wasting syndrome. A recombinant baculo-virus producing the protective ORF2 protein of PCV2 has recently become available as a vaccine for pigs [32].

\section{$>\quad$ Live viral vector vaccines}

Viral vectors are recombinant viruses that contain genetic material of the antigen of interest that will be delivered into cells. They represent an attractive tool to deliver and present vaccine antigens that may offer advantages over traditional platforms. Due to their ability to effectively induce both humoral and cell-mediated immune responses, viral vectors are deemed as an attractive alternative to the traditional platforms to deliver vaccine antigens [33]. Viral vector vaccines like DNA vaccines, carry DNA effectively into a host cell for production of antigenic proteins that can be tailored to stimulate a range of immune responses, including antibody (B cell), T helper cell (CD4+ T cell), and cytotoxic T lymphocyte (CTL, CD8+ T cell) mediated immunity [34].

Viral vector is the most effective means of gene transfer to modify specific cell type or tissue and can be manipulated to express therapeutic genes. They are, in general; delivered from live viruses but can be modified such as by deletion of genes that encode proteins involved viral replication or immuno modulation [35]. A number of virus families are under intensive development as vaccine 
vectors for human or veterinary uses. There are several viral vector vaccines that are currently in use for veterinary diseases. The approved vaccines include adenovirus, canarypox virus, fowlpox virus, attenuated yellow fever, and vaccinia virus vectors, all of which are relevant as potential human viral vectored vaccines [36]. The choice of virus for routine clinical use in gene therapy, virotherapy, and vaccine applications will depend on the efficiency of transgene expression (flexibility), ease of production, safety, toxicity and stability. For gene therapy to be successful, an appropriate amount of a therapeutic gene must be delivered into the target tissue without substantial toxicity [37].

The canarypox virus vector system ALVAC has been used as a platform for a range of veterinary vaccines including WNV, canine distemper virus, feline leukemia virus, rabies virus, and equine influenza virus [38]. Canarypox viruses and fowlpox viruses have the advantage of being more host restricted than vaccinia virus. While they produce an abortive infection in mammalian cells, canarypox virus recombinants still effectively express inserted foreign genes. Several veterinary viral vaccines have been produced using the ALVAC vector system [39].

Although advances in viral vector (Table 2) vaccines seem promising in providing effective immune response and for reducing the problems associated with RNA mutation, this technology does have limitations that include issue of pre-existing immunity or maternally derived immunity that interferes with the live vector itself and reduces the uptake of the antigen by the antigen presenting cells and consequently the transgene expression as well as specific immune response [40]. Low titer production especially in adeno-associated viruses, and lack of proper protein folding and glycosylation in the host system may alter the conformation and epitope arrangement that affect the immunogenicity and efficacy of the vaccine [31].

\begin{tabular}{|c|c|c|}
\hline Recombinant viral vector & Target pathogen & Target species \\
\hline \multirow{2}{*}{ Adenovirus } & Avian influenza virus & Poultry \\
\hline \multirow{2}{*}{ ALVAC (plus tetanus toxoid and carbopol adjuvant) } & Foot- and- mouth disease(FMD) & Horses \\
\hline \multirow{2}{*}{ Canarypox virus (ALVAC) } & Equine influenza virus & Horses \\
\cline { 2 - 3 } & West Nile Virus (WNV) & Horses \\
\cline { 2 - 3 } & Rabies virus & Cats \\
\cline { 2 - 3 } & Catine leukaemia virus (FeLV) & Dogs \\
\cline { 2 - 3 } & Canine distemper virus & Poultry \\
\hline \multirow{2}{*}{ Fowlpox virus (FPV) } & Avian influenza virus and FPV & Poultry \\
\hline Vaccinia virus & Newcastle disease virus (NDV) and FPV & Wildlife \\
\hline NDV (LaSota strain) & Rabies virus & Poultry \\
\hline Flavivirus YFV-17D (live chimeric virus) & Avian influenza virus and NDV & Horses \\
\hline Turkey Herpesvirus(HVT) (live chimeric virus) & IBDV and Marek's disease virus & Poultry \\
\hline
\end{tabular}

Table 2: Viral vector veterinary vaccines.

\section{$>\quad$ DNA vaccines}

DNA immunization is a novel technique used to efficiently stimulate humoral and cellular immune responses to protein antigens. DNA vaccines use a plasmid containing the gene(s) that code for an immunogenic protein(s) of interest. The recombinant plasmids containing a foreign gene are purified from the virus, and the "naked" DNA is injected directly into the animal, usually intramuscularly or intra-dermally (into the skin). The animal's cells take up the DNA, and an immune response is induced to the protein expressed from the foreign gene [41]. The direct injection of genetic material into a living host causes a small amount of its cells to produce the introduced gene products. This inappropriate gene expression within the host has important immunological consequences, resulting in the specific immune activation of the host against the gene delivered antigen [42].

The distinction between a sophisticated DNA vaccine and a simple viral vector may not be clear. Many aspects of the immune response generated by DNA vaccines are 
not understood. However, this has not impeded significant progress towards the use of this type of vaccine in humans and animals, and clinical trials have begun [43]. The last few years have seen the development of nucleic acid vaccines against many viral diseases like classical swine fever, rabies, foot and mouth disease, equine herpes infections, avian infectious bronchitis, avian influenza, infectious bursal disease and Newcastle disease (Table 3).

\begin{tabular}{|c|c|c|c|}
\hline Viral Disease & Host & Etiological agent & $\begin{array}{c}\text { Protective } \\
\text { Antigen Gene }\end{array}$ \\
\hline Bovine leukemia & Cattle & Retrovirus & gp51; gp30 \\
\hline Infectious bovine rhinotracheitis & Cattle & Bovine herpes virus & $\mathrm{gC} ; \mathrm{Gd}$ \\
\hline Bovine viral diarrhea & Cattle & Pestivirus & E2 \\
\hline Foot and mouth disease & Cattle & Picornavirus & VP1 \\
\hline Swine fever & Swine & Pestivirus & E2 \\
\hline Pseudorabies & Swine & Herpes virus & gB; gC; gD \\
\hline Parvoviral infections & Canines & Parvovirus & VP1; VP2 \\
\hline Rabies & Canines & Rhabdovirus & gp gene \\
\hline Canine distemper & Canines & Morbilivirus & HA; F \\
\hline Equine influenza & Equines & Influenza virus & $\mathrm{HA}$ \\
\hline Equine herpes infection & Equine & Herpes virus & gB; gC; gD \\
\hline Avian influenza & Poultry & Influenza virus & $\mathrm{HA}$ \\
\hline Newcastle disease & Poultry & Avian paramyxovirus & $\mathrm{HN} ; \mathrm{F}$ \\
\hline Infectious bronchitis & Poultry & Coronavirus & N; S1 \\
\hline Infectious bursal disease & Poultry & Avibirnavirus & VP2 \\
\hline Chicken infectious anemia & Poultry & Gyrovirus & VP1 and VP2 \\
\hline
\end{tabular}

Table 3: DNA viral vaccines for infectious diseases of Veterinary importance.

Source: [18].

In the host, native forms of the proteins have access to presentation pathways by class I Major Histocompatibility (MHC I) antigens in addition to class II MHC presentation, which results in a balanced immune response. The use of pure plasmid DNA offers many advantages over other vaccine delivery vehicles. One of the greatest advantages is the ability of DNA vaccines to induce both humeral and cell-mediated immune responses, which is critical for protection from many diseases. There is also evidence that DNA vaccines can induce long-term immunity, which is a further requirement for vaccine efficacy. As the vector itself does not induce immune responses, DNA vaccines can be repeatedly administered without the interference of antibodies [44].

From a technical viewpoint, DNA vaccines are easy to engineer, produce and purify. So, new DNA vaccines can be constructed and evaluated in animal models within months and large-scale production are available at costs considerably lower than traditional vaccines. DNA vaccines are very temperature stable and therefore have a long shelf life and can be transported without cold chain. The safety of DNA vaccines has been established in various trials in several species including humans. DNA vaccines encoding several antigens or proteins can be delivered to the host in a single dose, only requiring a microgram of plasmids to induce immune responses [45].

\section{Challenges in Veterinary Viral Vaccine Developments}

Biological and technical obstacles: The first set of difficulties is biological and technical in nature. Viruses have co-evolved/ coexist with their hosts, and have also developed many strategies to survive, in relative state of equilibrium, in a population or with a host. For example, they have acquired, often at the expense of their host, many molecules or mechanisms that enable them to withstand the various assaults of the immune response. This explains in part why, despite years of research, there is yet no vaccine available for some viral diseases [16].

Moreover, many viral infections, for example foot and mouth disease and avian influenza, are caused by pathogens that have several serotypes and which constantly evolve. A recent example of the problems 
brought about by serotypical variation is provided by the appearance of bluetongue in Northern Europe due to serotype 8 (one amongst the 24 known serotypes). Existing vaccines proved useless, as they did not contain the corresponding antigens. For vector-borne diseases such as bluetongue, vaccination seems to be the only solution, but it is very difficult to attack the vector, especially through methods that have no negative environmental impact [31].

Another difficulty inherent in veterinary vaccinology is the wide variety of target species, and the number of different infections in each one. Vaccines, as opposed to therapeutic molecules (antibiotics, anthelmintics), are for the most part extremely specific; there are only a few multi-species vaccines containing the same antigenic valence (tetanus, rabies etc.). Another problem for animal vaccination is that the target species include wildlife, which is not always easy to access for vaccination. For example, it is not possible to vaccinate vampire bats (Desmodusrotundus) in Latin America against rabies because of the difficulty in gaining access to these animals. Other wild species, however, such as foxes (Vulpesvulpes) (rabies) or wild boars (Susscrofa) (classical swine fever) are relatively easy to gain access to and can be vaccinated by means of vaccinal baits [44].

Economic obstacles: Other obstacles to vaccination are of an economic nature. The most important one is the low return on investment for private companies that develop veterinary vaccines for livestock, especially for diseases specific to developing countries, which unfortunately is where the needs are the greatest [16].

However, such problems are not confined to developing or transition countries, as certain target species get little attention even in developed countries if they are considered minor species. In Europe, for example, this is the case for milking sheep, goats, rabbits, and fish. In addition, these minor species are distributed unevenly over the different zones of Europe, which does not facilitate the implementation of European-wide procedures for marketing veterinary vaccines. This has led to the MUMS concept (Minor Use, Minor Species). Developing vaccines for these types of diseases requires an equitable public-private partnership [44].

Legal and regulatory obstacles: Another regulatory obstacle to the development of veterinary vaccines for livestock is the existence of very stringent and inflexible regulations governing the registration and marketing of veterinary drugs (including vaccines). Such legislation does not promote flexibility in the choice of vaccinal strains for vaccines capable of preventing infections caused by pathogens with multiple serotypes, or in their adaptation to the epidemiological conditions in the field. In addition, these very heavy regulations (which, however, present the considerable advantage of guaranteeing quality and efficacy against the pathogen involved, as well as the safety of commercial products) have recently been supplemented by additional regulations as a result of environmental impact studies for veterinary drugs (certain anthelmintics for example). Attenuated vaccines, however, require special attention, as they may become dispersed amongst the target population or infect other, non-target species in their vicinity [31].

Other obstacles: Public apprehension over vaccination or over certain products such as genetically modified organisms may constitute a final obstacle. A specific case of this occurred during the recent episode of FMD in the United Kingdom when it was found that consumers would have been reluctant to consume products from vaccinated animals. This mistrust was one of the (minor) reasons behind the decision not to resort to vaccination in attempting to control the disease in 2001 [16].

\section{Existing Opportunities for Veterinary Viral Vaccine Development}

Advancement in science and technology, together with improved knowledge in immunology, microbiology and recombinant technology has played pivotal roles in introducing novel ideas in vaccine [28]. Nowadays, recombinant, reassortant, or virus-like particles technologies open the road for new vaccines [46].

The field of DNA vaccination is developing rapidly. Vaccines currently being developed use not only DNA, but also include adjuncts that assist DNA to enter cells, target it towards specific cells, or that may act as adjuvants in stimulating or directing the immune response [43]. The availability of complete DNA sequences and a better understanding of gene function have allowed specific modifications or deletions to be introduced into the viral genome, with the aim of producing well-defined and stably attenuated live or inactivated viral vaccines [32].

An interesting development in genetically engineered viral vaccines is the production of chimera viruses that combine aspects of two infective viral genomes. A chimera PCV1-2 vaccine has the immunogenic capsid gene of PCV2 cloned into the backbone of the nonpathogenic PCV1 and induces protective immunity to 
wild-type PCV2 challenge in pigs [47]. A further sophistication of this approach is a recently developed vaccine against avian influenza virus, where the hemagglutinin (HA) gene has been removed from an H5N1 virus, inactivated by removing the polybasic amino acid sequences, and combined with the NA gene from an H2N3 virus onto an H1N1 "backbone" virus. A vaccine containing the resultant inactivated H5N3-expressing virus administered in a water-in-oil emulsion protects chickens and ducks against the highly pathogenic H5N1 strain [32].

Similarly, a live Flavivirus chimera vaccine against West Nile virus (WNV) in horses (PreveNile) was registered in the United States in 2006. In this chimera vaccine, the structural genes of the attenuated yellow fever YF-17D backbone virus have been replaced with structural genes of the related WNV. After a single shot, the vaccine stimulates both cell-mediated and humoral responses without causing any clinical illness or spreading to sentinel horses and provides protection against WNV challenge for up to 12 months (Preve Nile package insert). A similar vaccine could be a candidate for a human $W N V$ vaccine $[48,49]$.

\section{Production of Veterinary Viral Vaccines in Ethiopia}

The following table is summarized licensed veterinary viral vaccines in Ethiopia.

\begin{tabular}{|c|c|l|}
\hline S/N & Viral Vaccines & \multicolumn{1}{c|}{ Descriptions } \\
\hline 1 & LSD & Live attenuated vaccine containing Capripox virus strain cultured on VERO-cells \\
\hline 2 & Sheep \& goat Pox & Live freeze dried Capripox virus strain cultured on VERO-cells \\
\hline 3 & FMD & Bivalent vaccine containing 0, C, SAT1, SAT2 and A serotype \\
\hline 4 & PPR & Killed \\
\hline 5 & AHS & Monovalent/multivalent vaccine containing serotype 2, 4, 9 cultured on VERO cells \\
\hline 6 & Newcastle & Live \\
\hline 7 & Fowl Pox & $\begin{array}{l}\text { It is a live freeze dried viral vaccine produced on the Chicken Fibro blast cells of } \\
\text { embryonated specific pathogen free eggs using modified Fowl pox virus }\end{array}$ \\
\hline 8 & Gumboro & This vaccine contains infectious bursal disease virus intermediate standard strain. \\
\hline 9 & Camel pox & Live attenuated vaccine containing Camel Pox virus strain \\
\hline
\end{tabular}

Table 4: Licensed viral veterinary vaccines in Ethiopia.

\section{Conclusion and Recommendation}

Veterinary vaccine development starting from early Edward Jenner discovery, it has passed long routes. Veterinary vaccines have been providing a plenty of importance in controlling zoonotic and emerging disease as well as in food security. Vaccination can also improve animal production and reduce costs for sick animal treatment and risk of antibiotic residue. A continuous collaborate working between animal and human disease control agencies and scientists will be essential for reducing the ever-present danger of new, emerging diseases. Most vaccines are still based on live vaccines which are not generally desirable for commercial companies, because these vaccines have many limitations that make them uneconomical to produce. Developing recombinant technologies are the better opportunity for the future vaccine developments because they are feasible methods for mitigating the limitations faced by conventional vaccines. Even though many vaccines and vaccine producing technologies are available, several viral diseases have no vaccines yet. Many problems remain to be resolved to incorporate new knowledge and technologies into vaccine design. Especially in Ethiopia, there are several animal viral diseases that are affecting our livestock, but only few viral vaccines are being produced. This is seriously hindering us from getting the benefits that we should have get from animal productions.

Therefore, based on the above conclusion the following points are forwarded.

> There should be intimate collaboration between animal and human health professionals to keep their environment healthy that promote one health.

$>$ Novel vaccine technologies should be encouraged because can fill the limitations of conventional live and killed vaccines.

$>$ To be profitable from the veterinary viral vaccines the challenging factors for the development of the vaccines should be managed. 


\section{Open Access Journal of Veterinary Science \& Research}

All responsible animal health agencies should give emphasizes to the reduction of vaccine development obstacles.

> Particularly for Ethiopia, the issue of vaccine development should be given into special attention to control our livestock threating diseases.

\section{References}

1. Lambert (2005) Veterinary biologics and its development of gene therapy. General Medicine 2: 148-164.

2. OIE (2012) Terrestrial manual. Principle of veterinary vaccine production. Paris, pp: 45-51.

3. Zinkernagel RM (2000) Localisation dose and time of antigens determine immune reactivity. Semin Immunol 12(3): 163-171.

4. Milstien J, Kaddar M (2006) Managing the effect of TRIPS on availability of priority vaccines. Bull World Health Organ 84(5): 360-365.

5. Normile D (2008) Rinderpest. Driven to extinction. Science 319(5870): 1606-1609.

6. Basso M (2006) Intervention of health authorities in patent examination: the Brazilian practice of the prior consent. International Journal Intellect Property Manage 1(2): 5-74.

7. Heymann DL (2014) Ebola: learn from the past. Nature 514(7522): 299-300.

8. Brun A (2016) Vaccine technologies for veterinary viral diseases: Text book of Methods and protocols, CISA-INIA, Madrid, Springer, Spain, pp: 1.

9. Gutierrez AH, Spero DM, Gay C, Zimic M, De Groot AS (2012) New vaccines needed for pathogens infecting animals and humans: one Health. Human Vaccine Immunother 8(7): 971-978.

10. Monath TP (2013) Vaccines against diseases transmitted from animals to humans: a one health paradigm. Vaccine 31(46): 5321-5338.

11. Carvalho JA, Prazeres DM, Monteiro GA (2009) Bringing DNA vaccines closer to commercial use. I Drugs 12(10): 642-647.
12. Kumagai Y, Takeuchi O, Akira S (2008) TLR9 as a key receptor for the recognition of DNA. Adv Drug Deliv Rev 60(7): 795-804.

13. James M (2016) New Technologies in Veterinary Vaccine Development: BioVacc Consulting Ltd, Amersham, UK Presented at European Veterinary Vaccinology Workshop, Ghent, pp: 3.

14. FAO (2009) High-Level Expert Forum-How to Feed the World in 2050: Global agriculture towards 2050, Food and Agriculture Organization.

15. Roth JA (2011) Veterinary vaccines and their importance to animal health and public health. Procedia in Vaccinology 5: 127-136.

16. Pastoret P, Michael F, Brochier B (2007) Future trends in veterinary vaccinology. Research Gate 26(2): 489-494.

17. FAO (2010) Brucella melitensis in Eurasia and the Middle East, FAO Animal Production and Health Proceedings, Food and Agriculture Organization, pp: 10.

18. Dhama K, Mahendran M, Gupta PK, Rai A (2008) DNA vaccines and their applications in veterinary practice: current perspectives. Vet Res Commun 32(5): 341356.

19. Singer RS, Finch R, Wegener HC, Bywater R, Walters J, et al. (2003) Antibiotic resistance the interplay between antibiotic use in animals and human beings. The Lancet Infectious Diseases 3: 47-51.

20. WHO (2016) Why is vaccination important for addressing antibiotic resistance? World Health Organization.

21. Ward E (2006) Three-year vaccines: What, How and Why to tell your clients. In: Proceedings of North America Veterinary Conferences, Athens, Greek, 27: 789-798.

22. McVey S, Shi J (2010) Vaccines in veterinary medicine: a brief review of history and technology. Vet Clin North Am Small Anim Pract 40(3): 381-392.

23. Bergman JG, Muniz M, Sutton D, Fensome R, Ling F, et al. (2006) Comparative trial of the canine parvovirus, canine distemper virus and canine adenovirus type 2 fractions of two commercially available modified live vaccines. Vet Rec 159(22): 733-736. 


\section{Open Access Journal of Veterinary Science \& Research}

24. Capua I, Terregino C, Cattoli G, Toffan A (2004) Increased resistance of vaccinated turkeys to experimental infection with an H7N3 lowpathogenicity avian influenza virus. Avian Pathology 33(2): 158-163.

25. Uzzau S, Marogna G, Leori GS, Curtiss R, Schianchi G, et al. (2005) Virulence attenuation and live vaccine potential of aroA, crp cdt cya, and plasmid-cured mutants of Salmonella enterica serovar Abortusovis in mice and sheep. Infect Immun 73(7): 4302-4308.

26. Yang CD, Liao JT, Lai CY (2007) Induction of protective immunity in swine by recombinant bamboo mosaic virus expressing foot-and-mouth disease virus epitopes. BMC Biotechnoogyl 7(1): 62.

27. Bouzoubaa K, Kissi B, Kasmy M, Waddahou S, Tangarfi M (2005) Vaccination against Newcastle disease and Gumboro disease in backyard poultry: a pilot program in Morocco. In Abstract book of the 14th World Veterinary a Poultry Congress, Istanbul, Turkey. Abstract No: I3-314.

28. Babiuk LA (2003) Vaccination: a management tool in veterinary medicine. The Veterinary Journal 164(3): 188-201.

29. Toth K, Dhar D, Wold WS (2010) Oncolytic (replication-competent) adenoviruses as anticancer agents. Expert Opin Biol Ther 10(3): 353-368.

30. Ellis RW (2000) New technologies for making vaccines. Vaccine 17(13-14): 1596-1604.

31. Bande F, Arshad SS, Hair Bejo, M, Moeini H, Omar AR (2015) Progress and challenges toward the development of vaccines against avian infectious bronchitis. Journal of immunology research 2015(2015): 424860-424872.

32. Meeusen EN, Walker J, Peters A, Pastoret PP, Jungersen G (2007) Current status of veterinary vaccines. Clinical microbiology reviews 20(3): 489510.

33. Kumar P, Woon-Khiong C (2011) Optimization of lentiviral vectors generation for biomedical and clinical research purposes. Contemporary trends in technology development and applications. Gen Ther 11(2): 144-153.

34. Hanawa H, Yamamoto M, Zha H, Shimada T, Persons DA (2009) Optimized lentiviral vector design improves titer and transgene expression of vectors containing the chicken beta-globin locus HS4 insulator element. Mol Ther 17(4): 667-674.

35. Quakkelar ED, Redeker A, Haddad EK, Harari A, McCaughey SM, et al. (2011) Improved innate and adaptive immune stimulation by genetical modified HIV-1protein expressing NYVAC vectors. PLoS One 6: 16-81.

36. Draper SJ, Heeney JL (2010) Viruses as vaccine vectors for infectious diseases and cancer. Nature reviews Microbiology 8(1): 62-73.

37. Warnock JN, Daigre C, Al-Rubeai M (2011) Viral vectors for Gene therapy. Methods and protocols in molecular biology 737: 1-25.

38. Taylor J, Meignier B, Tartaglia J, Languet B, Vander Hoeven G, et al. (1995) Biological and immunogenic properties of a canarypox-rabies recombinant, ALVAC-RG (vCP65) in non-avian species. Vaccine 13(6): 539-549.

39. Minke JM, Audonnet JC, Fischer L (2004) Equine viral vaccines: the past, present and future. Vet Res 35(4): 425-448.

40. Faulkner OB, Estevez C, Yu Q, Suarez DL (2013) Passive antibody transfer in chickens to model maternal antibody after avian influenza vaccination. Veterinary Immunology and Immunopathology 152(3-4): 341-347.

41. Rodriguez F, Whitton JL (2000) Enhancing DNA immunization Virology 268(2): 233-238.

42. Koprowski H, Weiner DB (1998) DNA Vaccination/ Genetic Vaccination. Spriner-Verlag, Heidelberg 198.

43. World Health Organization (WHO) (2014) Biologicals.

44. Gurunathan S, Klinman DM, Seder RA (2000) DNA vaccines: immunology, application, and optimization. Annual review of immunology 18(1): 927-974.

45. Encke J, Jasper ZP, Jack R (1999) DNA Vaccines. Intervirology 42: 117-124.

46. Guerin N (2007) History of vaccination; from empiricism towards recombinant vaccine PubMed 28(1): 3-8. 
47. Fenaux M, Opriessnig T, Halbur PG, Elvinger F, Meng XJ (2004) A chimeric porcine circovirus (PCV) with the immunogenic capsid gene of the pathogenic PCV type 2 (PCV2) cloned into the genomic backbone of the non-pathogenic PCV1 induces protective immunity against PCV2 infection in pigs. Journal of Virology 78: 6297-6303.

48. Monath TP, Liu J, Kanesa-Thasan N, Myers GA, Nichols $\mathrm{R}$ et al. (2006) A live, attenuated recombinant West
Nile virus vaccine. Proc Natl Acad Sci USA 103(17): 6694-6699.

49. Ethiopia animal health yearbook (EAHYB) (2011) Ministry of Agriculture Animal and Plant Health Regulatory Directorate. October 2010 Addis Ababa, Ethiopia. 